\section{Kompass}

Dermatologie

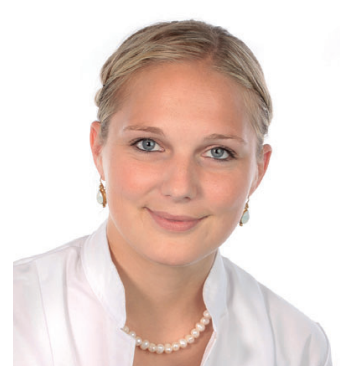

Saskia Schnabl-Scheu

Universitäts-Hautklinik Tübingen,

Tübingen, Deutschland

\title{
Saskia Schnabl-Scheu
}

\section{Gewinnerin des 2. Publikationspreises im Fachbereich Spezielle Dermatochirurgie 2019 der DGDC}

\section{Forschungsschwerpunkte}

- Lokale Infiltrationsanästhesie bei speziellen Indikationen in der Dermatochirurgie (Operation an den Akren, spezielle Operationen, spezielle Patientengruppen)

- Besonderheiten bei der Behandlung von seltenen Hauttumoren: Klinische, morphologische und histopathologische Aspekte

(1) Ausbreitungsverhalten und Prognose kutaner Plattenepithelkarzinome vom desmoplastischen Typ (dSCC)

(2) Ausbreitungsverhalten und Prognose kutaner Plattenepithelkarzinome an besonderen Lokalisationen

(3) Ausbreitungsverhalten und Prognose kutaner Sarkome

\section{Akademischer Werdegang}

- 01/2008 - 03/2010 Plastische Chirurgie Friedrich-Alexander-Universität Erlangen

- Seit 06/2010 Dermatologie, Schwerpunkt Dermatochirurgie, Universitäts-Hautklinik Tübingen

- 07/2015 Fachärztin für Haut- und Geschlechtskrankheiten

- 01/2018 Weiterbildungsprüfung Allergologie

- Seit 01/2020 Wissenschaftliche Freistellungsphase TÜFF Habilitandinnen Förderung der Universität Tübingen

Frau Dr. Schnabl-Scheu, was fasziniert Sie an der Dermatochirurgie?

Dr. Saskia Schnabl-Scheu: Mich fasziniert vor allem das breite Spektrum der Dermatochirurgie. Dabei meine ich nicht nur die Schwerpunktgebiete wie Tumorchirurgie, Phlebologie, Ästhetik mit Lasertherapie usw., sondern vor allem die Tumorchirurgie und ihre notwendigen Rekonstruktionen im Kopf-Halsbereich. Hier gibt es viele interdisziplinäre Grenzgebiete, die häufig eine spannende Zusammenarbeit bieten, zum Beispiel mit der Neurochirurgie, MKG, HNO, plastische Chirurgie, Pädiatrie oder Histologie/Pathologie, und einem die Möglichkeit geben, sich fachlich immer wieder weiterzuentwickeln.

Die Deutsche Gesellschaft für Dermatochirurgie hat Sie 2019 mit dem 2. Publikationspreis im Fachbereich «Spezielle Dermatochirurgie» ausgezeichnet - Fassen Sie für uns bitte kurz den Inhalt der Publikation zusammen.

Dr. Schnabl-Scheu: Kutane Sarkome sind selten und der pathogenetische Ursprung zeigt sich heterogen. In unserer Arbeit «Patterns of infiltration and local recurrences of different types of cutaneous sarcomas after 3D histology» (Anm. d. Red.: J Dtsch Dermatol Ges. 2018 
Dec;16(12):1434-1442) wurden Patienten mit Dermatofibrosarcoma protuberans, Myosarkomen, vaskulären Sarkomen und mit uneinheitlichen Sarkomentitäten, diverse dermale undifferenzierte pleomorphe Sarkome, bezüglich Infiltrationsmuster und Rezidivrate verglichen.

\section{Welche Erkenntnisse lassen sich für den Klinikalltag, für die Praxis mitnehmen?}

Dr. Schnabl-Scheu: Wichtig ist vor allem immer wieder hervorzuheben, dass man kutane Sarkome klar von Weichteilsarkomen differenzieren muss. Das ist in der aktuellen Literatur nur selten der Fall. Insgesamt besteht auf dem Gebiet der kutanen Sarkome noch viel Forschungsbedarf, um für die einzelnen Entitäten optimale diagnostische als auch therapeutische Richtlinien festzulegen. Die aktuelle Leitlinie zur Behandlung des Angiosarkoms ist aus dem Jahr 2012 und wird demnächst überarbeitet. Ein Treffen von Experten verschiedener Fachrichtungen zur Vorbereitung Anfang dieses Jahres hat erneut gezeigt, wie unterschiedlich die Therapieansätze in den einzelnen deutschen Kliniken bei diesem seltenen aber hoch aggressiven Krankheitsbild sind.

\section{Welche Themen gehören Ihrer Meinung nach zu den aktuellen «Hot Topics» in der Dermatochirurgie?}

Dr. Schnabl-Scheu: Hier gibt sicherlich viele «Hot Topics». Meiner Meinung nach stehen die Digitalisierung und deren Veränderungen für unser Fach ganz oben. Innovationen in der histologischen Datenverarbeitung kombiniert mit neuen Verfahren wie die Rapid Lump Examination (RLE) werden Abläufe beschleunigen, verändern und uns neue Möglichkeiten eröffnen.

Gibt es ein Thema, das Ihrer Meinung nach dringend zu einem «Hot Topic» werden sollte, ein Thema, das mehr Aufmerksamkeit verdient hätte?

Dr. Schnabl-Scheu: Aktuell hat die Dermatologie und damit auch die Dermatochirurgie noch nicht die gleichen Nachwuchsprobleme wie andere chirurgische Fachrichtungen. Aber wir sind ein Fach mit steigenden Patientenzahlen. Durch die zunehmende UV-Strahlung und die steigende Altersstruktur der Bevölkerung nehmen Operationszahlen bei Hautkrebs zu. Im Rahmen meiner Lehrleistung für die Habilitation betreue ich in einem sogenannten Mentorenprogramm Studenten ab dem 1. Semester. Von 10 Studenten sind 9 weiblich. Vor allem nach der Geburt von Kindern haben genau diese Frauen auch in meiner Fachrichtung einen Karriereknick, gerade wenn sie in Teilzeit arbeiten wollen. Aber auch die aktuellen
Ärztestreiks haben gezeigt, dass gerade bei den jüngeren Kollegen Work-Life-Balance einen hohen Stellenwert einnimmt. Auch männliche Kollegen nehmen ihre familiäre Verantwortung zunehmend wahr und gehen in Elternzeit. Es gibt immer mehr Paare mit Kindern die gerne beide reduziert, z.B. 80\%, arbeiten wollen, damit eine berufliche Chancengleichheit herrscht. Ich würde mir wünschen, dass es in Zukunft Modelle gibt, die das umsetzen. Das mag organisatorisch aufwendiger sein, zwei 50\% arbeitende Frauen oder Männer einzusetzen, als eine 100\% Telle zu besetzen, gerade in Führungspositionen, und als Klinik eine flexible, qualitativ hochwertige Kinderbetreuung zu gewährleisten. Dies wäre meiner Meinung nach aber eine Investition in die Zukunft. Attraktive Arbeitsplätze mit hoher Mitarbeiterzufriedenheit schaffen - die großen Wirtschaftsunternehmen haben das bereits verstanden und setzen es um, wenn sie gutes Personal halten wollen.

\section{Zum Abschluss noch zwei kurze, persönlichere Fragen: Der Alltag in} der Klinik ist sicher fordernd und öfter auch stressig. Worin finden Sie einen Ausgleich?

Dr. Schnabl-Scheu: Mein Ausgleich ist auf jeden Fall meine Familie. Meine zwei Kinder, 1 und 4 Jahre alt, lassen mich viele Dinge deutlich entspannter sehen. Ich habe als Jugendliche Leistungssport gemacht und merke auch heute noch, dass ich ein geregeltes Pensum an Sport für meine Ausgeglichenheit brauche. Meistens kommen mir dabei auch die besten Ideen.

\section{Und schließlich: Verraten Sie uns, wofür Sie das Preisgeld verwendet haben?}

Dr. Schnabl-Scheu: Hochrangige Journals verlangen mittlerweile Bearbeitungs- und Publikationsgebühren. Einen Teil des Geldes habe ich zur Einreichung eines Papers beim Journal of Investigative Dermatology (JID) verwendet, dass dort mittlerweile akzeptiert wurde (Anm. d. Red.: Haug et al., Prognostic impact of perineural invasion in cutaneous squamous cell carcinoma. Results of a prospective study of 1399 tumors). Außerdem habe ich mit einem Kollegen aus unserer dermatohistologischen Abteilung ein neues Projekt zum Dermatofibrosarkoma protuberans. Der Rest des Preisgeldes ist zur Durchführung dieses Projekts eingeplant.

Frau Dr. Schnabl-Scheu, ich danke Ihnen für das Gespräch und wünsche natürlich alles Gute!

Das Interview führte unser Redakteur Christoph Habel 